\title{
TDP-43 M337V Mutation in Familial Amyotrophic Lateral Sclerosis in Japan
}

\author{
Akira Tamaoka ${ }^{1}$, Makoto $\mathrm{Arai}^{2}$, Masanari Itokawa ${ }^{2}$, Tetsuaki $\mathrm{Arai}^{2}$, Masato Hasegawa ${ }^{2}$, \\ Kuniaki Tsuchiya ${ }^{3}$, Hiroshi Takuma ${ }^{1}$, Hiroshi Tsuji ${ }^{1}$, Akiko Ishii ${ }^{1}$, Masahiko Watanabe ${ }^{1}$, \\ Yuji Takahashi ${ }^{4}$, Jun Goto ${ }^{4}$, Shoji Tsuji ${ }^{4}$ and Haruhiko Akiyama ${ }^{2}$
}

\begin{abstract}
The clinical features of a Japanese family with autosomal dominant adult-onset amyotrophic lateral sclerosis (ALS) are reported. Weakness initially affected the bulbar musculature, with later involvement of the extremities. Genetic studies failed to detect any mutations of the $\mathrm{Cu} / \mathrm{Zn}$ superoxide dismutase-1 (SOD1) and Dynactin1 (DCTN1) genes, but revealed a single base pair change from wild-type adenine to guanine at position 1009 in TAR-DNA-binding protein (TDP-43), resulting in a methionine-to-valine substitution at position 337. The immunohistochemical study on autopsied brain of the proband's aunt showed TDP-43-positive cytoplasmic inclusions in the anterior horn cells of the spinal cord and in the hypoglossal nucleus, as well as glial cytoplasmic inclusions in the precentral gyrus, suggesting that a neuroglial proteinopathy was related to TDP-43. In conclusion, a characteristic clinical phenotype of familial ALS with initial bulbar symptoms occurred in this family with TDP-43 M337V substitution, the pathomechanism of which should be elucidated.
\end{abstract}

Key words: Amyotrophic lateral sclerosis (ALS), TAR-DNA-binding protein 43 (TDP-43)

(Inter Med 49: 331-334, 2010)

(DOI: 10.2169/internalmedicine.49.2915)

\section{Introduction}

Amyotrophic lateral sclerosis (ALS) is a progressive and fatal neurodegenerative disorder that is characterized pathologically by the degeneration of motor neurons in the brain and spinal cord, and clinically by progressive weakness and death within a few years of onset. Recently, TAR DNA-binding protein 43 (TDP-43) was identified as the major pathological protein in the motor neuron inclusions found in sporadic ALS and superoxide dismutase 1 (SOD1)negative familial ALS, as well as in frontotemporal lobar degeneration with ubiquitin-immunoreactive, tau-negative inclusions (FTLD-U). Although the role of TDP-43 in the pathogenesis of these neurodegenerative disorders remains to be elucidated, several mutations of TDP-43 have been identified in individuals with sporadic and familial ALS, sug- gesting that TDP-43 may be a causative protein for these disorders (1-6). Here we first report the detailed clinical features of affected members of a Japanese family who suffered from ALS linked to TDP-43 M337V mutation.

\section{Case Report}

The proband (III-2 in Fig. 1-1) was a Japanese woman aged 61 years. She developed dysarthria at the age of 55 years, which became progressively worse. One year later, she also noted dysphagia. Neurological examination at the age of 56 revealed minimal atrophy of the facial muscles and tongue, markedly diminished reflexes of the palatal and pharyngeal muscles, and slow movements and minimal fasciculation of the tongue. Her deep tendon reflexes, including the jaw jerk, were highly exaggerated. At the age of 57, her dysphagia worsened, and atrophy and fasciculation of the

\footnotetext{
${ }^{1}$ Department of Neurology, Doctoral Program in Medical Sciences for Control of Pathological Processes, Graduate School of Comprehensive Human Sciences, University of Tsukuba, Tsukuba, ${ }^{2}$ Tokyo Institute of Psychiatry, Tokyo, ${ }^{3}$ Tokyo Metropolitan Matsuzawa Hospital, Tokyo and ${ }^{4}$ Department of Neurology, Graduate School of Medicine, University of Tokyo, Tokyo

Received for publication September 18, 2009; Accepted for publication October 18, 2009

Correspondence to Dr. Akira Tamaoka, atamaoka@md.tsukuba.ac.jp
} 
$1-1$

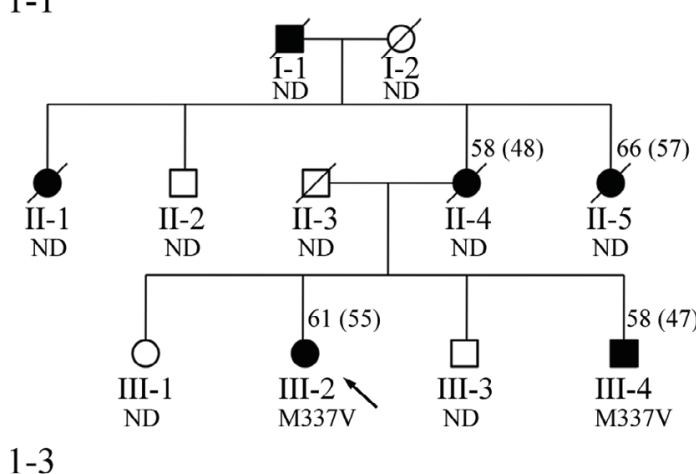

$1-2$ c.1009A>G (Met337Val)
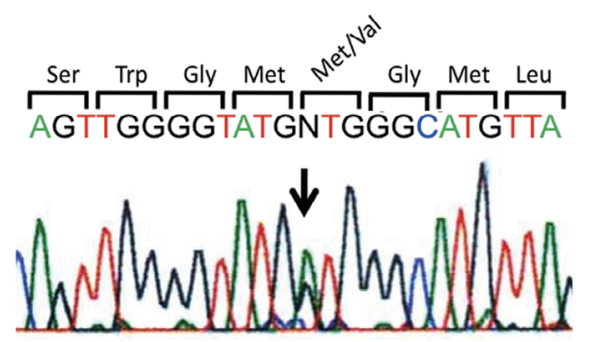
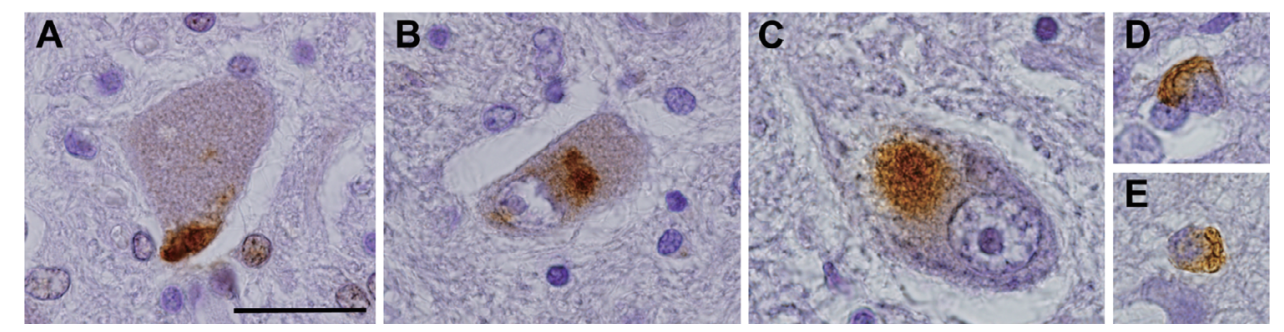

Figure 1. 1-1. Pedigree of the present family. Circles represent women and squares represent men. The slashed symbols indicate deceased subjects. Known affected persons are shown as filled symbols. The arrow represents the proband. Age at death or current age and age at disease onset in parenthesis are indicated. ND=not determined. 1-2. Chromatogram of Patient III-2 (the proband). Chromatogram shows the heterozygous sequence trace of $A$ to $G$ for genotyping by the reverse primer. The nucleotide position of substitution is indicated by arrow. 1-3. Immunocytochemical findings in Patient II-5. TDP-43 positive cytoplasmic inclusions in the anterior horn of the spinal cord (A, B) and in the hypoglossal nucleus (C). Glial cytoplasmic inclusions in the precentral gyrus (D, E). (A, C) Phosphorylation-independent anti-TDP-43 antibody; (B, D, E) phosphorylationdependent anti-TDP-43 antibody (pS409/410). The sections were counterstained with hematoxylin to reveal nuclei. Bar in $\mathbf{A}=\mathbf{2 5} \mu \mathrm{m}$.

tongue became more prominent. Muscle weakness of the lower extremities showed slow progression, predominantly in the distal regions. At the age of 58, she was almost unable to protrude her tongue. At the age of 61, she also noted mild weakness of the upper extremities. Needle EMG showed marked neurogenic changes of the biceps, abducens pollicis brevis, vastus lateralis and tibialis anterior muscles of the right side, as well as a mild neurogenic pattern in her right masseter.

The aunt of the proband (II-5 in Fig. 1-1), a Japanese woman, developed dysarthria at the age of 57 years, followed by dysphagia, weakness of the upper extremities, and difficulty with breathing. She could walk without support until her death at the age of 66 . The results of the neuropathological examination were reported in detail (7).

The younger brother of the proband (III-4 in Fig. 1-1), a Japanese man, developed dysarthiria at the age of 44 years. Neurological examination at the age of 47 showed slight dysarthria, poor movement of the soft palate, exaggerated pharyngeal reflexes and jaw jerk, slow movements, slight atrophy and fasciculation of the tongue. These findings were mainly related to pseudobulbar palsy. He also showed hyperreflexia in the upper and lower extremities (predominantly in the latter) without any pathological reflexes. Nee- dle EMG revealed neurogenic changes of the masseter and orbicularis oris muscles, while there was a normal pattern in the tongue and extremities. He had no dysphagia, muscle weakness, or atrophy of the upper and lower extremities, as well as no sensory disturbance or vesicorectal disturbance. He could stand and walk unaided. His condition deteriorated slowly and progressively over the next 10 years. At present, he is 58 years old and virtually bed-ridden with a gastrostomy and minimal communication. Patient II-4, Patient II-1 and Patient I-1 all suffered from dysarthria until death, the details of which were unknown.

The present family demonstrated autosomal dominant inheretance of ALS and both sexes were affected. Six family members (patients I-1, II-1, II-4, II-5, III-2 and III-4) were suspected to have ALS, among whom three (II-5, III-2 and III4) had definite ALS according to the El-Escorial criteria. All six patients ( 2 men and 4 women) with familial ALS in this family showed dysarthria at the onset, so their clinical courses were indistinguishable from bulbar-onset ALS. There was no history of dementia and no atypical features in the kindred. Based on the information of the patients with good clinical records (patients II-4, II-5, III-2 and III-4), the mean age of symptom onset was 52.5 years (range 44-61 years) and the mean disease duration was 9.5 years (range 
9-10 years) from symptom onset to death based on the outcome in patients II-4 and II-5.

After approval by the Ethics Committees of all participating institutions, sequencing of the coding regions of the TDP-43 gene in the patients (III-2 and III-4) was performed, which showed a heterozygous A-to-G transition at cDNA position 1009 (c.1009A $>\mathrm{G})$ resulting in a methione-to-valine substitution at position 337 (M337V) in a highly conserved region of exon 6 (Fig. 1-2). None of the control 1,621 healthy subjects providing informed consent had this missense mutation.

Immunohistochemistry analysis of the brain of patient II-5 using both a phosphorylation-independent anti-TDP-43 antibody (10782-2-AP) and a phosphorylation-dependent antiTDP-43 antibody (pS409/410) (8) showed neuronal cytoplasmic inclusions in the anterior horn of the spinal cord (Fig. 1-3A, B) and the hypoglossal nucleus (Fig. 1-3C), as well as glial cytoplasmic inclusions in the precentral gyrus (Fig. 1-3D, E).

\section{Discussion}

In the present study, we detected the M337V substitution in TDP-43 in a Japanese family with ALS, including one case confirmed at autopsy (patient II-5). We consider that this M337V substitution was associated with the disease, since M337V was present in two affected individuals from one generation and never in the control subjects, in addition to the fact that M337V substitution of TDP-43 has already been reported to segregate with ALS within two probably unrelated kindreds $(2,6)$. In a UK autosomal dominant ALS family carrying M337V substitution of TDP-43 reported by Sreedharan et al (2), three had limb-onset ALS and two had bulbar-onset ALS. The mean age of symptom onset was 47 years (range 44 to 52). Mean disease duration was 5.5 years (range 4 to 7) from symptom onset to death. The M337V mutation carrier in a US family with a strong family history of ALS reported by Rutherford et al (6) showed upper limbonset ALS at 38 years of age, 6 years younger than the earliest onset age reported in the British M337V family (2). In the present paper, we show the first Japanese family with ALS carrying M337V substition of TDP-43, in which virtually all patients showed dysarthria at the onset, suggesting that their clinical courses were indistinguishable from bulbar-onset ALS. Among these UK, US and Japanese families carrying TDP-43 M337V mutation, the common features include no signs of dementia or other atypical features of ALS and past middle age onset of the disease. However, the signs at onset were different among these three families, and mean disease duration in the present Japanese family was longer than that in the UK family, indicating the phenotype of this mutation is quite variable. The identification of $\mathrm{M} 337 \mathrm{~V}$ in three genealogically unrelated ALS families further implies the pathogenicity of TDP-43 M337V mutation.

Regarding the pathogenecity of TDP-43 M337V mutation, Sreedharan et al (2) reported that mutant forms of TDP-43 (including $\mathrm{M} 337 \mathrm{~V}$ ) fragmented in vitro more easily than wild-type TDP-43 and, in vivo, caused neuronal apoptosis and developmental delay in chick embryos, suggesting a pathophysiological link between TDP-43 and ALS. In addition, Rutherford et al (6) showed that biochemical analysis of TDP-43 in lymphoblastoid cell lines of carriers with TDP-43 mutations including M337V revealed a substantial increase in fragments possibly cleaved by caspase, including the $\sim 25 \mathrm{kDa}$ fragment, compared to control cell lines, supporting TDA-43 as a cause of ALS. Our immunohistochemical study showed TDP-43 positive cytoplasmic inclusions in the anterior horn cells of the spinal cord and in the hypoglossal nucleus, as well as glial cytoplasmic inclusions in the precentral gyrus, suggesting that a neuroglial proteinopathy was related to TDP-43. Further investigations including biochemical analysis using patients' fibroblasts or lymphoblastoid cells will be necessary to elucidate the mechanism by which TDP-43 contributes to ALS and to develop new drugs that block the pathological process related to TDP-43.

\section{Acknowledgement}

The authors thank Dr. Shuzo Shintani, Department of Neurology, Toride Kyodo Hospital and Dr. Kazuo Yoshizawa, Department of Neurology, National Hospital Organization Mito Medical Center for their clinical information on some patients of this family. This work was supported in part by grants from Ministry of Health, Labor, and Welfare, Japan and Ministry of Education, Culture, Science and Technology, Japan.

\section{References}

1. Gitcho MA, Baloh RH, Chakraverty S, et al. TDP-43 A315T mutation in familial motor neuron disease. Ann Neurol 63: 535-538, 2008.

2. Sreedharan J, Blair IP, Tripathi VB, et al. TDP-43 mutations in familial and sporadic amyotrophic lateral sclerosis. Science 319: 1668-1672, 2008.

3. Yokoseki A, Shiga A, Tan CF, et al. TDP-43 mutation in familial amyotrophic lateral sclerosis. Ann Neurol 63: 538-542, 2008.

4. Kabashi E, Valdmanis PN, Dion $P$, et al. TARDBP mutations in individuals with sporadic and familial amyotrophic lateral sclerosis. Nat Genet 40: 572-574, 2008.
5. Van Deerlin VM, Leverenz JB, Bekris LM, et al. TARDBP mutations in amyotrophic lateral sclerosis with TDP-43 neuropathology: a genetic and histopathological analysis. Lancet Neurol 7: 409-416, 2008.

6. Rutherford NJ, Zhang YJ, Baker M, et al. Novel mutations in TARDBP (TDP-43) in patients with familial amyotrophic lateral sclerosis. PLoS Genetics 4: e1000193, 2008.

7. Tsuchiya K, Shintani S, Nakabayashi H, et al. Familial amyotrophic lateral sclerosis with onset in bulbar sign, benign clinical course, and Bunina bodies: a clinical, genetic, and pathological study of a Japanese family. Acta Neuropathol 100: 603- 
Inter Med 49: 331-334, 2010 DOI: 10.2169/internalmedicine.49.2915

607, 2000.

8. Hasegawa M, Arai T, Nonaka T, et al. Phosphorylated TDP-43 in frontotemporal lobar degeneration and amyotrophic lateral sclerosis. Ann Neurol 64: 60-70, 2008.

(C) 2010 The Japanese Society of Internal Medicine http://www.naika.or.jp/imindex.html 